\title{
Feedback of the behaviour of a silo founded on a compressible soil improved by floating stone columns
}

\author{
Ramdane Bahar ${ }^{1}$, Omar Sadaoui ${ }^{2}$ and Fatma Zohra Yagoub ${ }^{3}$ \\ ${ }^{1}$ USTHB, Laboratoire Eau, Environnement, Géomécanique et Ouvrages, Faculté de Génie Civil, Bab-Ezzouar, Algérie \\ ${ }^{2}$ Université Abderrahmane Mira de Bejaia, Département Génie Civil, Bejaia, Algérie \\ ${ }^{3}$ Direction de projets, Groupe Cevital Spa, Bejaia, Algérie
}

\begin{abstract}
The coastal city of Bejaia, located 250 kilometers east of the capital Algiers, Algeria, is characterized by soft soils. The residual grounds encountered on the first 40 meters usually have a low bearing capacity, high compressibility, insufficient strength, and subject to the risk of liquefaction. These unfavorable soil conditions require deep foundations or soil improvement. Since late 1990s, stone columns technique is used to improve the weak soils of the harbor area of the city. A shallow raft foundation on soft soil improved by stone columns was designed for a heavy storage steel silo and two towers. The improvement of $18 \mathrm{~m}$ depth have not reached the substratum located at $39 \mathrm{~m}$ depth. The stresses transmitted to the service limit state are variable 73 to $376 \mathrm{kPa}$. A rigorous and ongoing monitoring of the evolution of loads in the silo and settlements of the soil was carried out during 1400 days that is from the construction of foundations in 2008 to 2012. After the loading of the silo in 2010, settlement occurred affecting the stability of the towers due to excessive differential settlements. Consequently, the towers were inclined and damaged the transporter. This paper presents and discusses the experience feedback of the behavior of these structures. Numerical calculations by finite elements have been carried and the results are compared with the measurements.
\end{abstract}

\section{Introduction}

La ville côtière de Bejaia est située à 250 kilomètres à l'est de la capitale Alger, en Algérie. L'expansion urbaine et le développement industriel de la région à partir des années 2000 ont engendré l'occupation des terrains proches de la zone portuaire de la ville. Ces terrains sont constitués de formations d'âge quaternaire, et les reconnaissances géotechniques ont permis de les caractériser dans la catégorie de sols marécageux inondables de faible portance, très compressibles et sujets au risque de liquéfaction [1-2].

En raison des conditions du sol défavorables, la majorité des ouvrages fondés sur des fondations superficielles ont subi des dommages structuraux inhérents aux tassements excessifs nécessitant un renforcement supplémentaire par des travaux de reprise en sous-œuvre très coûteux pour exploiter à nouveau ces structures en toute sécurité [2-3]. Devant ce constat, l'expansion des activités industrielles à partir des années 2000 avait impulsé les concepteurs de projets et les maîtres d'ouvrages à adopter soit des fondations profondes sur pieux fichés dans le substratum, soit de procéder au renforcement des sols pour l'adoption de semelles superficielles.
Parmi les procédés de renforcement de sols, la technique de colonnes ballastées offre une alternative intéressante $\mathrm{du}$ fait qu'elle utilise le ballast comme matériau de substitution disponible localement. Ce procédé économique et de réalisation rapide contribue à l'augmentation de la portance des sols médiocres, réduit les tassements et les risques de liquéfaction des sables saturés [4-9]. Cette technique de renforcement a été appliquée pour la première fois en Algérie en 1999 dans le traitement des sols mous sous les silos de stockage de céréales au port de Bejaia.

Après la réalisation de certains ouvrages lourds, une instrumentation a été mise en place en vue de suivre l'évolution des tassements dans le temps en fonction des charges appliquées. Les mesures enregistrées ont dépassé la prévision initiale au stade de projet. Ce qui s'est traduit par interactions entre ouvrages, des pathologies de fondations et des dommages structuraux importants ayant engendré des arrêts d'exploitation silos [10].

L'article traite du retour d'expérience en termes de tassements et de pathologies des fondations d'un silo de stockage de 80000 tonnes de capacité et de deux (02) tours d'ensilage fondés sur un sol compressible renforcé par des colonnes ballastées flottantes. 


\section{Site expérimental et description des ouvrages}

Le site d'étude est situé à l'embouchure d'oued Soummam et à proximité du port de Bejaia, il s'étend sur une superficie d'environ 8 hectares. La majeure partie des terrains ont été gagnés sur la mer, ils sont sujets à l'influence des courants marins et les fluctuations de la nappe phréatique.

Les ouvrages de stockage ont été conçus pour augmenter les capacités d'emmagasinement en sucre blanc. Ils rentrent dans le cadre du développement des activités du groupe Cevital SPa en matière de traitement et de conditionnement de sucre d'une capacité de 3000 t/j. Les installations constituent l'une des plus importantes en Afrique en termes de hauteur et de capacité de stockage. La superstructure du silo 80000 tonnes de capacité est conçue en charpente métallique, de $54.3 \mathrm{~m}$ de diamètre intérieur, de $63 \mathrm{~m}$ de hauteur et de poids à vide de l'ordre de 3000 tonnes (Fig. 1). La coque et le toit sont constitués d'une paroi cylindrique sous forme d'assemblage en double viroles en acier. D'autre part, le radier rigide en béton armé de $58 \mathrm{~m}$ de diamètre est fondé sur un gros béton de $0.75 \mathrm{~m}$ d'épaisseur, il est constitué de nervures orthogonales $(0.7 \mathrm{~m} / 3 \mathrm{~m})$ et d'une plaque de $0.5 \mathrm{~m}$ d'épaisseur. La contrainte due au poids propre est de 73 $\mathrm{kPa}$. La contrainte maximale résultant de la descente des charges à l'état limite de service (ELS) transmise sous le radier est de $376 \mathrm{kPa}$. Concernant les tours d'ensilage 1 et 2, leurs contraintes en service (ELS) sont respectivement de 242 et $200 \mathrm{kPa}$. Le sol est renforcé par des colonnes ballastées de $18 \mathrm{~m}$ de longueur, réparties uniformément suivant un maillage de $1.6 \times 1.6\left(\mathrm{~m}^{2}\right)$ et n'ont pas atteint le substratum situé à $39 \mathrm{~m}$ de profondeur. A l'achèvement des ouvrages en 2009, une instrumentation (tassomètres, cibles optiques, capteurs de déformation) a été mise en œuvre avant la mise en chargement du silo survenu en avril 2010.

\section{Contextes géologique et géotechnique}

Les formations géologiques rencontrées jusqu'à $47 \mathrm{~m}$ de profondeur sont d'âge quaternaire, elles surmontent la couche marno- calcaire dure du crétacé supérieur qu'on retrouve entre 45 et $50 \mathrm{~m}$ de profondeur [11]. La formation quaternaire est constituée principalement de dépôts d'alluvions marécageuses de nature argileuse et sableuse lâches et imprégnées d'une matrice vaseuse (Fig. 2). Les investigations géotechniques dénotent une faible résistance du sol au cisaillement et une forte compressibilité, cela est confirmé par certains paramètres mécaniques mesurés sur échantillons intacts. Tenant compte du faible taux de consolidation et de la présence de la nappe et des courants marins, le sol a un comportement évolutif.

L'analyse granulométrique montre que 60 à $95 \%$ des passants sont inférieurs à $80 \mu \mathrm{m}$, ce qui dénote une constitution fine de la majorité des couches de sols. En outre, les essais de limites d'Atterberg ont permis de classifier ces sols dans la catégorie de plastique à très plastique. Le sol est très compressible et présente une tendance à la liquéfaction sur quelques lentilles sablonneuses. Les essais de cisaillement drainés (CD) ont révélé une faible résistance au cisaillement des sols quaternaires $\left(10 \leq \mathrm{c}^{\prime} \leq 42 \mathrm{kPa}\right.$ et $\left.5 \leq \varphi^{\prime} \leq 25^{\circ}\right)$, les valeurs moyennes varient de 18 à $25 \mathrm{kPa}$ pour la cohésion c' et de 10 à $15^{\circ}$ pour le frottement interne $\varphi^{\prime}$. Les paramètres mesurés dans la marne entre 39 et $47 \mathrm{~m}$ de profondeur varient dans les intervalles $\left(12 \leq \mathrm{c}^{\prime} \leq 52 \mathrm{kPa}\right.$ et $17 \leq \varphi^{\prime} \leq$ $33^{\circ}$ ), ce qui dénote une bonne résistance. Les essais oedométriques entre 3.5 et $39 \mathrm{~m}$ de profondeur ont donné un coefficient de compression $\mathrm{c}_{\mathrm{c}}$ variable de 0.09 à 0.27 et un coefficient de gonflement $\mathrm{c}_{\mathrm{g}}$ entre 0.01 et 0.08 . La formation quaternaire est sous à normalement consolidée et le substratum est surconsolidé à partir de $50 \mathrm{~m}$ de profondeur. Le niveau moyen de la nappe phréatique est à $-2 \mathrm{~m} / \mathrm{NGAL}$.

\section{Suivi des tassements}

Les relevés de l'évolution des chargements du silo et des tassements durant 1400 jours ont été effectués d'une manière continue depuis 2008 à 2012. Les tassements ont été mesurés sur 17 points notés $(\mathrm{T} 1, \ldots \ldots$. à $\mathrm{T} 17)$ par des tassomètres et des visées topographiques sur réflecteurs fixes (Fig. 3). L'incrément de contrainte de 73 à $260 \mathrm{kPa}$ sous l'effet d'une charge d'exploitation de l'ordre de 50000 tonnes (60\% de la charge maximale) a engendré à partir 2012 des tassements absolus variables de 44 à $53 \mathrm{~cm}$ sous le silo et de 27 à $30 \mathrm{~cm}$ respectivement sous les tours 2 et 1 (Fig. 4). Les tassements différentiels y afférent varient de 5 à $8 \mathrm{~cm}$ sous le silo et de 8 à $18 \mathrm{~cm}$ respectivement sous les tours 1 et 2 (Fig. 5). Les premiers indices pathologiques se manifestèrent à partir de février 2010. Ils sont inhérents aux tassements différentiels ayant impacté la stabilité des tours et des locaux techniques. Il s'agit de l'endommagement des transporteurs reliant la tour 1 au silo, de fissures béantes des murs des locaux techniques et l'inclinaison des tours 1 et 2 . L'inclinaison des tours a été décelée par des mesures topographiques, un déplacement horizontal maximal au sommet variant de 42 à $51 \mathrm{~cm}$ a été mesuré le mois de novembre 2010 .

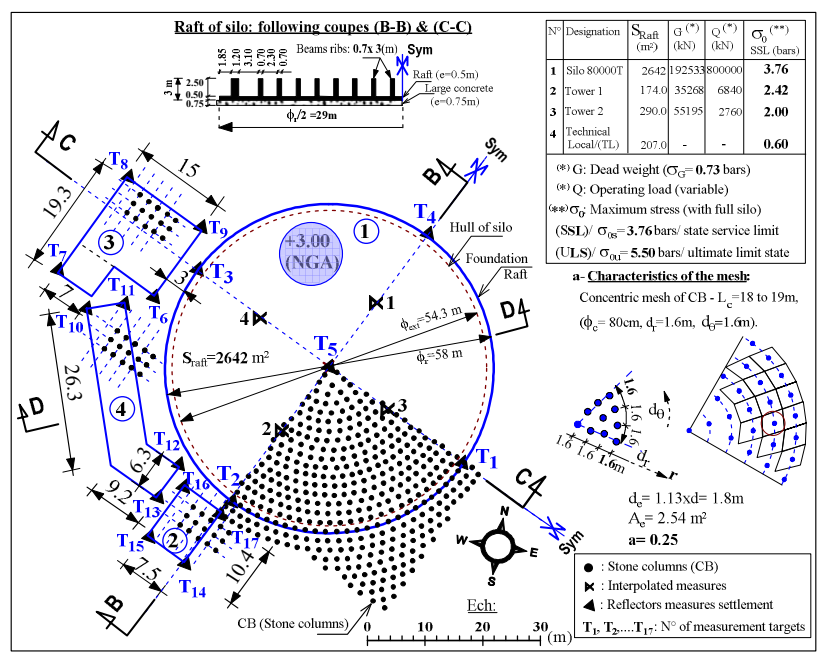

Fig.1. Site expérimental (Géométrie, descente de charges, maillage en $\mathrm{CB}$, ....etc). 


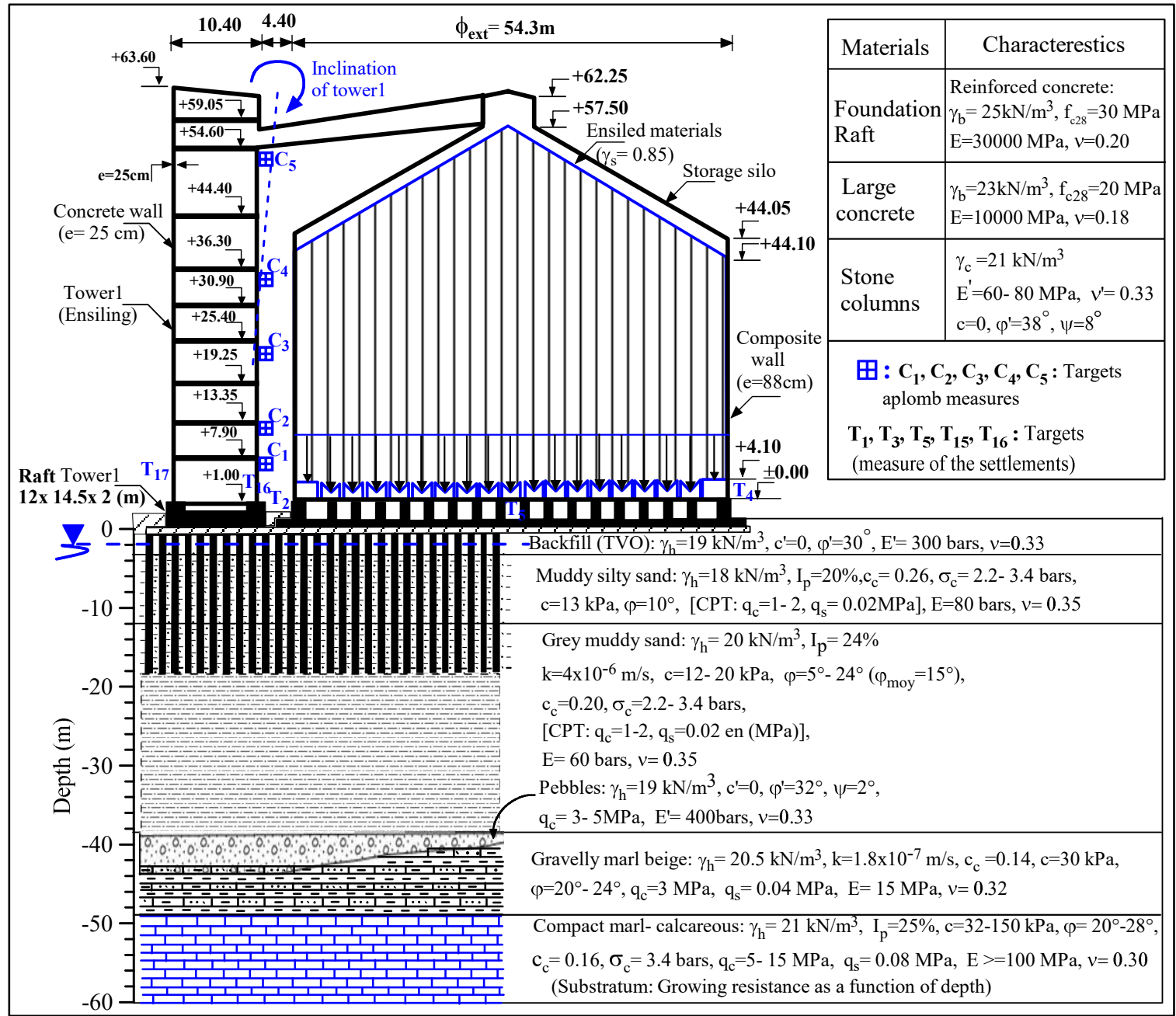

Fig. 2. Profil géotechnique au droit du site expérimental.

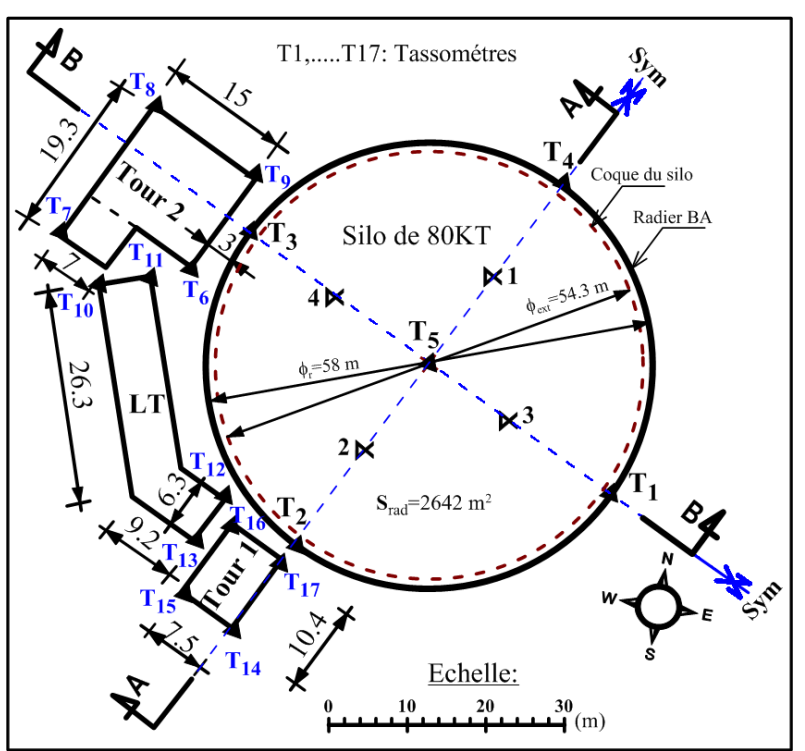

Fig. 3. Vue en plan et dispositifs de mesure des tassements.

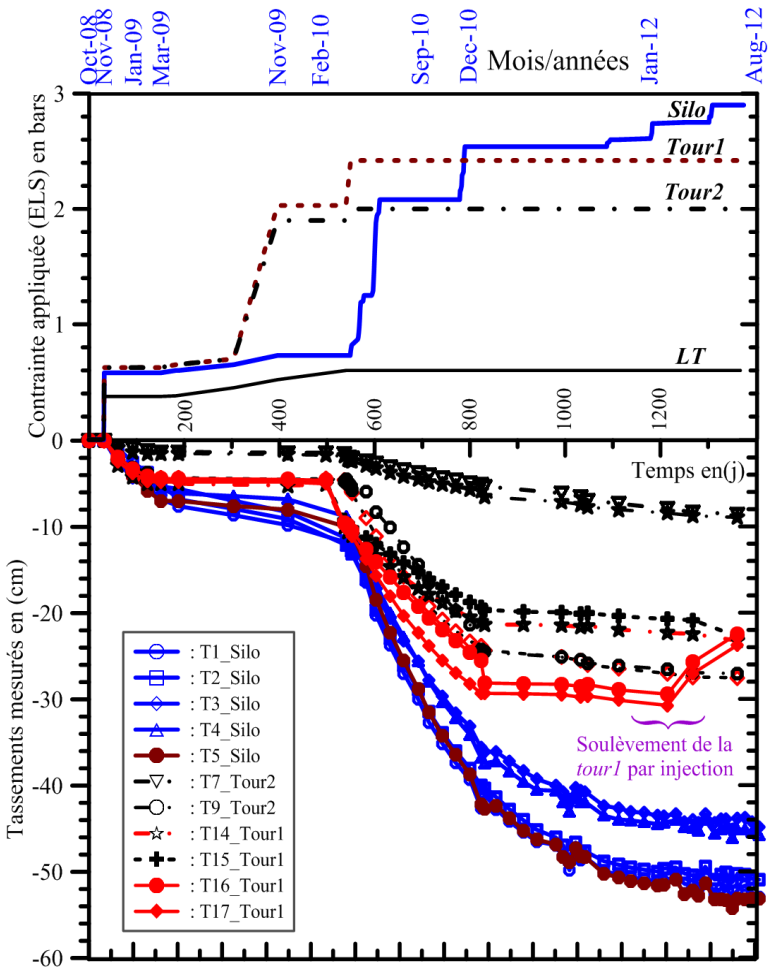

Fig. 4. Evolution des tassements en fonction du temps. 


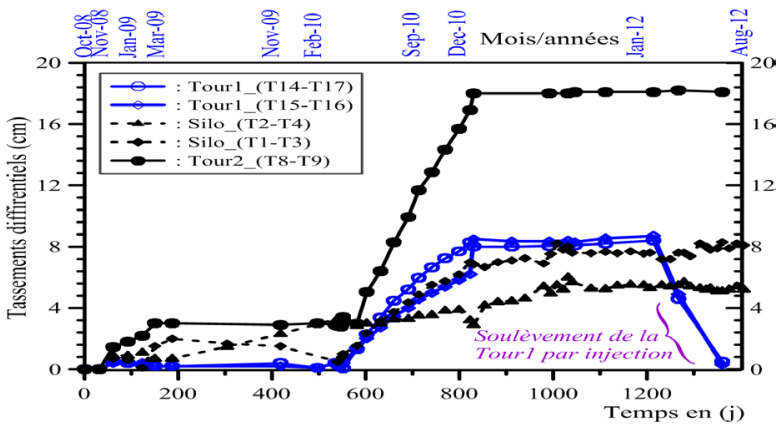

Fig. 5. Evolution des tassements différentiels.

\section{Calcul numérique}

Les calculs numériques ont été réalisés à l'aide du code d'éléments finis Cesar- LCPC [12] en considérant une loi de comportement élastoplastique avec le critère de MohrCoulomb. Le modèle tridimensionnelle (3D) prend en compte $3 / 4$ du radier du silo en intégrant les radiers des tours et les locaux techniques (LT). Il est constitué de 12930 éléments iso-paramétriques hexaédriques H8 (Fig. 6). Les chargements on été appliqués suivant le phasage de la construction des ouvrages et de l'ensilagedéchargement du silo de stockage (Fig. 4). La rigidité (EI) des radiers du silo et des tours est de l'ordre de $34 \mathrm{GPa} . \mathrm{m}^{2} / \mathrm{m}$ et celle du radier des locaux techniques est de $2.5 \mathrm{GPa} . \mathrm{m}^{2} / \mathrm{m}$. Les paramètres géomécaniques des couches de sol sont indiqués dans le tableau 1 et ceux de la couche renforcée (0-18m) ont été évalués par la méthode d'homogénéisation de Priebe [13]. Les calculs ont été menés par phasage suivant les contraintes réelles apportées par les ouvrages comme indiqué dans la Figure 4.

Tableau 1. Caractéristiques physiques et mécaniques prises en compte dans le modèle numérique.

\begin{tabular}{|l|c|c|c|c|c|c|}
\hline $\begin{array}{l}\text { Type de sols et des } \\
\text { matériaux }\end{array}$ & $\begin{array}{c}\mathrm{EP} \\
(\mathrm{m})\end{array}$ & $\begin{array}{c}\gamma \\
\left(\mathrm{kN} / \mathrm{m}^{3}\right)\end{array}$ & $\begin{array}{c}\mathrm{c}^{\prime} \\
(\mathrm{KPa})\end{array}$ & $\varphi^{\prime}\left(^{\circ}\right)$ & $\begin{array}{c}\mathrm{E}_{\mathrm{s}} \\
(\mathrm{MPa})\end{array}$ & $v^{\prime}$ \\
\hline $\begin{array}{l}\text { Remblai argilo- } \\
\text { caillouteux }\end{array}$ & 3.5 & 19 & 1 & 30 & 20 & 0.33 \\
\hline $\begin{array}{l}\text { Alluvions } \\
\text { compressibles }\end{array}$ & 35.5 & 19 & 18 & 13 & $6-8$ & 0.35 \\
\hline Marne graveleuse & 8.0 & 20.5 & 30 & 23 & 15 & 0.32 \\
\hline $\begin{array}{l}\text { Marne grise } \\
\text { compacte (Crétacé) }\end{array}$ & $\geq 28$ & 21 & $50-100$ & $25-30$ & $10^{2}-10^{3}$ & 0.30 \\
\hline Colonnes ballastées & 19 & 21 & 1 & 38 & $60-80$ & 0.33 \\
\hline Radier en BA RN30 & - & 25 & - & - & $3 \times 10^{4}$ & 0.20 \\
\hline
\end{tabular}

Les résultats des calculs numériques par éléments finis de la Figure 7 donnent un tassement maximal de 70 $\mathrm{cm}$ sous le centre du radier, soit un écart de $30 \%$ par rapport aux mesures. Cet écart est imputable à la variabilité des paramètres géotechniques et aux choix des modules de déformation des sols. Néanmoins, on remarque que l'évolution des cuvettes de tassements mesurés est concordante par rapport aux calculs numériques. Le coefficient de réduction des tassements $(\beta)$ est un paramètre régissant l'efficacité du traitement, il s'agit du rapport des tassements de consolidation sans et avec renforcement du sol. D'après les calculs à partir de la méthode de Priebe [13] et des mesures, ce paramètre varie de 1.25 à 1.30 dans notre cas.

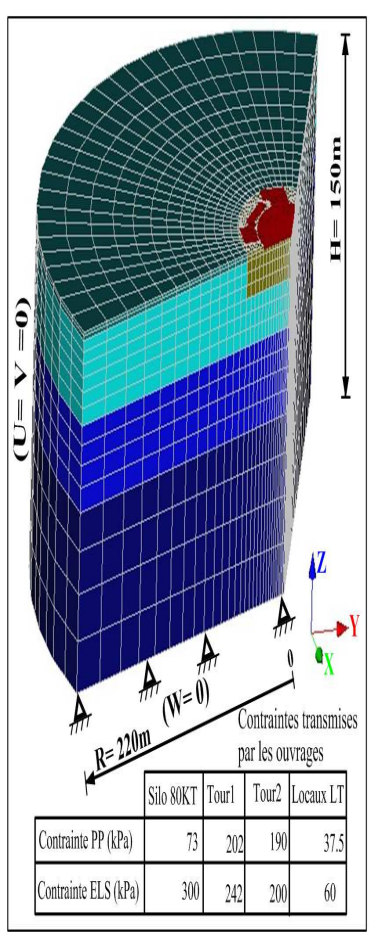

Fig.6. Modèle éléments finis tridimensionnelle à l'aide du logiciel Cesar- LCPC

Distance suivant profil (A.A) $(\mathrm{m})$

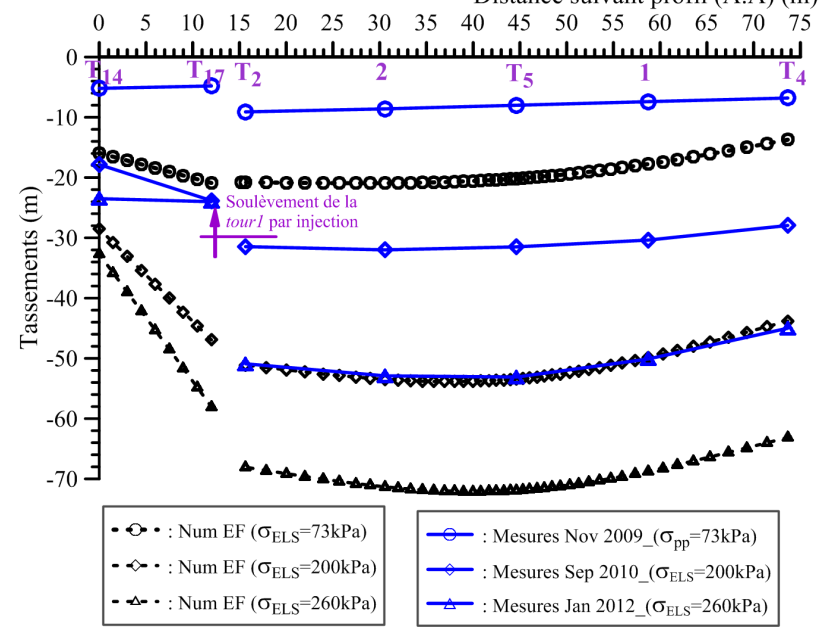

Fig. 7. Evolution de la cuvette des tassements des radiers. 


\section{Conclusion}

La présente étude met en évidence l'inefficacité d'un renforcement par colonnes ballastées flottantes dans des sols mous soumis à l'interaction d'ouvrages à chargement variable. La prévision des tassements au stade de projet était correcte et proche des mesures enregistrées. L'erreur commise dans l'appréciation des effets d'un tassement absolus dépassant les $50 \mathrm{~cm}$ est très coûteuse. En dépit de la rigidité élevée des radiers conçus pour pallier aux risques déflexions, ils ont accusé des tassements différentiels excessifs qui sont à l'origine de l'instabilité des tours fondées sur des radiers rigides de faibles surfaces $\left(174\right.$ à $290 \mathrm{~m}^{2}$ ). A noter également le caractère rapide du développement des tassements à cause l'accélération du drainage dû à la forte perméabilité des colonnes ballastées. Tenant compte des risques d'évolution des tassements, la capacité de stockage du silo prévue initialement à 80000 tonnes, n'a pas dépassé $62 \%$ depuis 2012 à ce jour.

\section{Remerciements}

Nous remercions le personnel technique de la direction projets Cevital SPa Bejaia et les techniciens d'exploitation de la raffinerie 3000T pour leurs contributions dans l'opération d'instrumentation de mesure des tassements et de relevé des chargements du silo. Nous remercions vivement également les cadres techniques du Bureau d'engineering des travaux publics BICS pour la mise à disposition de la station de surveillance topographique et leur assistance technique.

\section{Références}

1. M. Khiatine, L. Saci, R. Bahar, O. Sadaoui, Evaluation of dynamics properties and liquefaction potentiel of silty sand in harbour of Bejaia, Algeria. Proc. 2nd European Conf. on Earthquake Geotechnical Engineering and Seismology, Istambul- Turkey, pp. 650-659 (2013).

2. R. Bahar, O. Sadaoui, D. Amzal, Diffirenital settlements of cylindrical steel storage tanks- Case of the marine terminal of Bejaia. 7th Int. Conf. on case Histories in Geotechnical Engineering, Chicago USA, Paper 12, http://scholarsmine.mst.edu/7icchge/session02/12 (2013).

3. R. Bahar, M. Khiatine, O. Belhassani, O. Sadaoui, Settlements observations of industrial structures founded on soft soils: Case of the harbour zone of Bejaia city. ,. Proc. 3rd Int. Conf. on new Developments in Soils Mechanics and Geotechnical Engineering, Nocosia, North Cyprus, pp. 149-156 (2012).

4. R.E. Brown, Vibroflotation compaction of cohesionless soils. ASCE Journal of the Geotechnical Engineering Division 103 (12),. pp. 1435-1451 (1977).

5. H. B Seed, R.J. Booker, Stabilization of potentially liquefiable sand deposits using gravel drains. ASCE
Journal of Geotechnical Engineering Division 103 (7),. pp. 757-768 (1977).

6. H.J. Priebe, The prevention of liquefaction by vibroreplacement. Proc., Earthquake Resistance Construction and Design, Berlin, Germany (1989).

7. K. Adalier, A. El Gamal . Mitigation of liquefaction and associated ground deformations by stone columns. Engineering Geology, 72 (1),. pp. 678-690 (2004).

8. A. Dhouib, F. Blondeau 2005. Colonnes ballastées: Techniques de mise en oeuvre, domaines d'application, comportement, justification, contrôle, axes de recherche et de developpement. Presses de l'Ecole Nationale des ponts et Chaussées (ENPC), Paris (2005).

9. S. Lambert. Évaluation de la réduction du risque de liquéfaction par des colonnes ballastées. Proceedings of the 18th International Conference on Soil Mechanics and Geotechnical Engineering, Paris 2013. pp. 1-4 (2013).

10. O. Sadaoui, R. Bahar, Field measurements and back calculations of settlements of structures founded on improved soft soils by stone columns, European Journal of Environmental and Civil Engineering, http://dx.doi.org/10.1080/19648189.2016.1271358 (2017)

11. R. Bahar, O. Sadaoui, E. Vincens 2010. Settlements of silos founded on compressible soisl reinforced by stone columns. Proceedings of International Geotechnical Conference- Geotechnical Challenges in Megacities, Moscou (Russie), 07- 10juin 2010, Vol.3. pp. 965-970 (2010).

12. Itech 2004. Manuel de référence de Cesar et Solveur CLEO 2D \& 3D.

13. H.J. Priebe, The design of vibro- remplacement. Reprinted from: Ground Engineering . Keller Grundbau GmbH, Technical paper 12- 61E. 16pages. 1995 (1995). 